\title{
Caracterización de personalidad de mujeres adolescentes infractoras de ley: un estudio comparativo ${ }^{1}$
}

\author{
Eugenia Vinet ${ }^{2}$ \\ Paula Alarcón Bañares \\ Universidad de La Frontera, Temuco, Chile
}

\begin{abstract}
Resumen: El objetivo del presente estudio fue caracterizar a un grupo de adolescentes infractoras de ley diferenciándolas de varones infractores y de niñas adolescentes con problemas clínicos. Participaron 90 adolescentes de 13 a 18 años pertenecientes a tres grupos: mujeres infractoras, hombres infractores y mujeres con problemas clínicos. Ellos fueron evaluados con el Inventario Clínico para Adolescentes de Millon (MACI) y la Ficha de Escalada y Riesgo (FER) usando un diseño descriptivo correlacional. Se encontró que las adolescentes infractoras cometen menos delitos y de menor gravedad que los varones, pero presentan más condiciones de riesgo. Su perfil de personalidad muestra un estilo trasgresor activo, compatible con el de los varones y alteraciones emocionales más graves que las encontradas en las adolescentes con manifestaciones clínicas. El perfil obtenido es congruente con los antecedentes internacionales sobre delincuencia juvenil y relevante para orientar programas de intervención especializada para adolescentes infractoras de ley.
\end{abstract}

Palabras clave: delincuencia juvenil, mujeres, personalidad.

\section{Caracterização da personalidade de adolescentes do sexo feminino em conflito com a lei: um estudo comparativo}

\begin{abstract}
Resumo: O objetivo deste estudo foi caracterizar um grupo de adolescentes do sexo feminino infratores da lei enfatizando suas diferenças em relação a jovens do sexo masculino infratores e meninas com problemas clínicos. Participaram 90 adolescentes, entre 13 e 18 anos, pertencentes a três grupos: meninas infratoras, meninos infratores e meninas com problemas clínicos. Os participantes foram avaliados conforme o Inventário Clínico para Adolescentes de Millon (MACI) e Ficha de Escalada e Risco (FER), a partir de um desenho descritivo correlacional. Os resultados indicam que as adolescentes infratoras cometem menos delitos e de menor gravidade que os jovens, mas apresentam maiores condições de risco. O seu perfil de personalidade mostra um estilo transgressor ativo, igual ao encontrado nos jovens meninos, e alterações emocionais mais graves que as encontradas nas adolescentes com manifestações clínicas. Este perfil é compatível com os antecedentes internacionais sobre delinquência juvenil e relevante para orientar a intervenção especializada.
\end{abstract}

Palavras-chave: delinquência juvenil, mulheres, personalidade.

\section{Personality characterization of female juvenile offenders: a comparative study}

\begin{abstract}
This study aimed to characterize a group of female juvenile offenders emphasizing differences from male and female juvenile offenders with clinical problems. Participants were 90 adolescents from 13 to 18 years old, who belonged to three groups: female juvenile offenders, male juvenile offenders and female with clinical problems. Participants were evaluated through the Millon Adolescent Clinical Inventory (MACI) and the Escalation and Risk Record Card (FER) using a descriptive correlational design. Results showed that females perpetrated fewer and less severe offences than did males; however, they present more risk factors. The female personality profile revealed an active, unruly style, similar to males' and more severe emotional disorders than those found in females with clinical problems. This female personality profile is congruent with international studies about juvenile delinquency and relevant for guiding specialized intervention programs for female young offenders.
\end{abstract}

Keywords: juvenile delinquency, women, personality.

Al igual que muchos otros ámbitos de la Psicología, el estudio psicológico de la delincuencia ha sido criticado por poner escasa atención en las mujeres y poseer un sesgo interpretativo androcéntrico cuando la delincuencia femenina ha sido estudiada (Stefurak \& Calhoun, 2007). Sin embargo, la delincuencia femenina ha aumentado considerablemente en la última década y está ocurriendo en edades cada vez más tempranas (Mullis, Cornille, Mullis, \& Huber, 2004), siendo

1 Esta investigación recibió financiamiento de la Dirección de Investigación de la Universidad de La Frontera a través del Proyecto 120612.

2 Endereço para correspondência:

Prof. Dra. Eugenia Vinet. Universidad de La Frontera. Facultad de Educación y Humanidades. Departamento de Psicología. Avenida Francisco Salazar 01145. Temuco, Chile. Casilla Postal 54-D. E-mail: evinet@ufro.cl relevante el estudio de las mujeres adolescentes infractoras de ley pues las investigaciones informan que se ha producido un cambio cuantitativo y cualitativo en los patrones de comportamiento violento y antisocial en los adolescentes que afecta especialmente a la población femenina (Odgers e cols., 2007).

Este es un segmento de la población poco estudiado en Psicología, tanto por su escasa presencia como porque la mayoría de los estudios sobre conducta delictiva en adolescentes se ha desarrollado en muestras de adolescentes varones. En general, se plantea que las mujeres adolescentes infractoras de ley se caracterizan por su "invisibilidad" pues "son tan pocas que no cuentan" (Navarro, 2005, p. 296) y porque se encuentran en una situación de especial vulnerabilidad y discriminación al confluir en ellas las condiciones 
de ser mujer, ser pobre, haber violado la ley y tener menos de 18 años (Juliano, 2008). Este estudio busca realizar una caracterización de adolescentes mujeres que realizan comportamientos antisociales resaltando, desde una perspectiva descriptiva, las diferencias en sus necesidades psicológicas al compararlas con adolescentes infractores varones y con adolescentes mujeres que presentan problemas clínicos no externalizados en desadaptación social. El énfasis descriptivo de la investigación no pretende realizar un análisis desde una perspectiva de género, sino dar cuenta de una receptividad psicológica y social diferenciada de este grupo de adolescentes infractoras.

\section{Salud mental de las adolescentes infractoras}

Entre las características personales de riesgo de las adolescentes infractoras de ley se señala que, en general, son jóvenes entre 14 y 16 años que se desarrollan en sectores urbanos pobres y con alta delincuencia; presentan mal desempeño académico y abandonan el colegio; abusan de alcohol y drogas y tienen necesidades médicas y de salud mental no cubiertas; experimentando sentimientos de opresión y carencia de esperanza en el futuro (Mullis e cols., 2004). Ellas tienen necesidades diferentes de las de sus pares masculinos pues con frecuencia han sufrido experiencias de maltrato, abuso infantil y explotación, muchas veces perpetradas por familiares cercanos; muchas de ellas serán jefas de hogar solteras con todas las implicaciones de pobreza y dificultades parentales que ello conlleva; presentan baja autoestima, con alta incidencia de conductas suicidas (Lenssen, Doreleijers, Van Dijk, \& Hartman, 2000; McCabe, Lansing, Garland, \& Hough, 2002). Además, se reporta que presentan mayor prevalencia de trastornos mentales en general $-84 \%$ con respecto al $27 \%$ registrado en sus pares varones - (Timmons-Mitchell e cols., 1997), particularmente en los trastornos mentales internalizados (Andrade, Silva, \& Assumpção, 2004).

El menoscabo en la salud mental de las adolescentes, se vincula a la afectación por trastorno de estrés postraumático, victimización por violencia intrafamiliar $\mathrm{y}$ alteraciones del comportamiento que acompañan el abuso de substancias (Dixon, Howie, \& Starling, 2004). Así mismo, el contexto familiar aparece como un factor de gran impacto en el comportamiento antisocial de las adolescentes, tanto desde la perspectiva del riesgo como desde su rol protector. En un estudio con 50 adolescentes brasileñas que cumplían sanciones judiciales por infracciones de ley se detectan, como experiencias de alto impacto, eventos estresantes en el dominio familiar como no recibir cuidado y atención de sus padres, no poder conocerlos o bien la muerte de uno de ellos o de un hermano (Dell'Aglio, Benetti, Deretti, Bergesch, \& Severo, 2005). Por el contrario, la percepción de la adolescente de cohesión y apego familiar aparece en diferentes estudios como una variable minimizadora de los comportamientos disruptivos y delictivos actuando como un importante factor protector (Cruise, Marsee, Dandreaux, \&
DePrato, 2007; Musitu, Buelga, Lila, \& Cava, 2001; Brasil, Alves, Amparo, \& Frajorge, 2006).

Diferentes variables pueden intervenir en su diferenciación con respecto al comportamiento delictivo masculino. Se plantea que las jóvenes tienen mayor control social reduciendo su permanencia en la calle, se enfrentan menos a la autoridad, sus pares las validan por conductas menos competitivas, riesgosas y no necesitan adoptar una identidad delictiva para protegerse en sectores de alto vandalismo. Destaca el hecho de que ellas desarrollan mecanismos de protección diferentes a los desarrollados por los varones al fortalecer los vínculos de apego con sus familias y vecinos (Emler \& Reicher, 1995). Sin embargo, este hecho conlleva una mayor exposición a las dinámicas familiares y permite comprender la mayor afectación psicológica, por experiencias traumáticas, abusivas y violentas al interior de la familia, descritas anteriormente en las adolescentes infractoras de ley. A esta mayor exposición, se suma la posibilidad de embarazo y maternidad temprana, experiencia que puede intervenir como factor de protección deteniendo la trayectoria delictiva o, a la inversa, intensifica el riesgo potencial para la dupla madre e hijo (Dell'Aglio e cols., 2005). En relación a las manifestaciones delictivas de las adolescentes, se señala que los delitos son menos violentos que los cometidos por adolescentes varones y que incluyen, con mayor frecuencia, desajustes conductuales leves que no son considerados delitos en los adultos (abandono del hogar, violaciones de los horarios y reglas establecidas en el hogar); además, algunos delitos como el robo en tiendas y la prostitución son considerados estereotipadamente femeninos (Mullis e cols., 2004).

En Chile, las cifras de jóvenes detenidas oscilan entre el $8 \%$ y el $16 \%$ del total de adolescentes detenidos (Servicio Nacional de Menores, 2007). Por su parte, las descripciones sobre características de las jóvenes delincuentes suelen coincidir con la literatura internacional. Se destaca que existen diferencias en los procesos de socialización según género, las niñas son mucho más controladas en sus vidas por los padres, más propensas a infligirse heridas, a la depresión y a desórdenes alimenticios; además, se reitera que tienen menor probabilidad que los hombres de involucrarse en delitos violentos (Corporación Chilena Pro Derechos de los Niños y los Jóvenes, 2006).

\section{EI MACI en la evaluación de personalidad de las adolescentes infractoras}

En los estudios sobre personalidad de adolescentes infractores también se presenta el fenómeno de invisibilidad femenina pues la gran mayoría de las investigaciones que consideran estas variables están realizadas en muestras de varones. Entre estos estudios destacan los desarrollados con el Inventario Clínico para Adolescentes de Millon (MACI) (Millon, 1993) el cual ha sido muy utilizado para estudiar variables de personalidad asociadas a la delincuencia juvenil.

El MACI es un autoinforme de construcción teórica, que 
evalúa estilos de personalidad, preocupaciones psicológicas y psicopatología en adolescentes. Los estilos de personalidad son formas de funcionamiento psicológico que surgen a través del desarrollo infantil y se estabilizan en la adolescencia como precursores de los estilos de personalidad adulta. Las preocupaciones psicológicas se relacionan con aspectos significativos del contexto de desarrollo del adolescente que pueden causarle preocupación, incluyen aspectos vinculados al desarrollo de la identidad, la corporalidad, la sexualidad y las relaciones sociales con los pares, la familia y la sociedad mayor. El área psicopatológica incluye los trastornos afectivos y de la adaptación social que tienen mayor prevalencia en la adolescencia.

El MACI ha tenido un especial desarrollo en la Psicología chilena. Se cuenta con una versión nacional (Vinet e cols., 1999) que posee buenas características psicométricas en adolescentes de población general y con trastornos psicopatológicos (Vinet \& Alarcón, 2003) y una capacidad adecuada para caracterizar a diversos grupos de adolescentes, entre ellos a adolescentes con problemas de desadaptación social (Alarcón, Vinet, \& Salvo, 2005). Además, la versión chilena del MACI posee normas propias con baremos diferenciados por sexo para adolescentes entre 13 y 19 años (Vinet \& Forns, 2008).

Entre los estudios desarrollados con el MACI en adolescentes infractores de ley existen algunos que han trabajado con muestras de hombres y mujeres, aunque muy pocos han explorado diferencias según género. Uno de ellos es el estudio de Loper, Hoffschmidt y Ash (2001) realizado en Estados Unidos en una muestra mixta (42 mujeres y 40 hombres) que había cometido delitos violentos, el otro estudio es de Alarcón (2001) realizado con adolescentes chilenos.

Loper e cols. (2001) indican que no se encontraron diferencias según genero en las características de los delitos ni en la reacción de los y las adolescentes frente a ellos, pero que escalas de los tres ámbitos de evaluación del MACI presentaron diferencias significativas. Las adolescentes mujeres presentaron, con respecto a los varones, rasgos de mayor introversión, autodegradación y personalidad límite; más autodevaluación, disconformidad con el propio cuerpo, sentimientos de inseguridad en relación a sus iguales y preocupaciones en relación a abuso infantil; también presentaron mayor probabilidad de cuadros clínicos como trastornos alimentarios, afecto depresivo y tendencia suicida. Esta información permite concluir a Loper e cols. (2001) que, aún en casos caracterizados por conductas delictivas externalizadas, las adolescentes evidencian más experiencias disfóricas asociadas a trastornos internalizados que los varones.

Alarcón (2001) caracterizó a un grupo de adolescentes infractores de ley compuesto por 73 hombres y 31 mujeres. $\mathrm{Al}$ trabajar con los puntajes directos de las escalas, esta autora observó diferencias significativas en 19 de las 27 escalas clínicas del MACI. En cuanto a estilos de la personalidad, las mujeres reportaron ser más introvertidas, inhibidas, afligidas, autodegradantes y más inestables emocionalmente que los varones, presentando funcionamiento límite con mayor frecuencia. Sus preocupaciones más relevantes indicaron devaluación personal, desaprobación del propio cuerpo, sentimientos de inseguridad grupal, percepción de dinámicas familiares alteradas que las afectan y sensación de daño por experiencias de abusos en la infancia. Los síndromes clínicos más prevalentes indicaron la posibilidad de trastornos de la alimentación y patología emocional que se expresa en sentimientos ansiosos, depresivos y riesgo de conductas autodestructivas. Alarcón (2001) señala que hombres y mujeres presentaron una acentuación de los patrones Trasgresor, Oposicionista y Poderoso junto a la Tendencia al Abuso de Sustancias y Tendencia a la Impulsividad indicando que estos estilos están asociados a la conducta de desadaptación social en adolescentes, características que serían comunes a los dos sexos.

Un estudio tipológico realizado recientemente por Stefurak y Calhoun (2007) exploró la presencia de subtipos en una muestra de adolescentes con transgresiones de ley y estado psiquiátrico moderadamente severo, susceptibles de ser tratadas con intervenciones ambulatorias. Al utilizar un análisis de conglomerados a partir de las escalas del MACI estas autoras determinaron una solución óptima de tres agrupaciones: Tipo 1 o "Problemas Externalizados"; Tipo 2 o "Depresivas Interpersonalmente Ambivalentes"; Tipo 3 o "Ansiosas Prosociales".

El Tipo 1 sugiere una tendencia activa y disruptiva hacia las relaciones sociales y gratificación de necesidades con presencia de problemas externalizados. El Tipo 2 plantea problemas significativos con la familia y la posibilidad de experiencias de abuso que pueden manifestarse en trastornos depresivos y riesgo suicida. El Tipo 3 sugiere un estilo interpersonal caracterizado por conductas emocionales, con una autoestima elevada asociada a una alta dependencia interpersonal, preocupación por las reglas y las expectativas de los otros.

Stefurak y Calhoun (2007) señalan que el Tipo 1 parece ser el que mejor calza con las trayectorias de desarrollo delictivo identificadas por Silverthorn y Frick (1999). El Tipo 2 parece representar el prototipo de mujeres delincuentes juveniles descrito en la criminología de orientación feminista donde destacan las historias de victimización y problemas emocionales de sus integrantes. El Tipo 3 parece representar a delincuentes ocasionales que se ven involucradas en conductas delictivas leves como parte de su pasaje por la adolescencia como etapa del desarrollo, las cuales también han sido descritas en la literatura especializada (Moffitt, 1993).

\section{Objetivos}

Los antecedentes expuestos revelan, consistentemente, el compromiso de variables psicológicas en el comportamiento delictivo de las adolescentes, señalando un foco importante para la evaluación diagnóstica y la detección de necesidades diferenciales específicas. Atendiendo a estas 
caracterizaciones y al estado del desarrollo del MACI en Chile, en el presente estudio se busca caracterizar a un grupo de adolescentes chilenas infractoras de ley utilizando las normas de este instrumento recientemente desarrolladas (Vinet \& Forns, 2008). El perfil del MACI de las jóvenes delincuentes será comparado con el perfil obtenido por un grupo similar de varones infractores de ley con objeto de revisar las diferencias de género descritas en la literatura; además, el mismo perfil se comparará con el perfil obtenido por un grupo de adolescentes mujeres con problemas de salud mental con el objeto de precisar las características más exclusivas de las jóvenes delincuentes que pudieran ser de particular relevancia para la intervención. Detectar perfiles de personalidad y necesidades psicológicas asociadas al comportamiento delictual femenino durante la adolescencia permitirá avanzar en programas de intervención y reinserción especializados para este grupo de riesgo.

Así, el objetivo de este estudio es doble. Se espera superar, en parte, la carencia de estudios con adolescentes mujeres infractoras de ley al dedicarles especial atención comparándolas con sus pares varones y también con otras adolescentes que presentan problemas psicológicos pero no en el ámbito de la desadaptación social. Adicionalmente, se evaluará la adecuación de los baremos chilenos del MACI (Vinet \& Forns, 2008) para el trabajo con estos grupos poblacionales, estableciendo su comparabilidad y continuidad con los estudios internacionales expresados en puntajes Tasa Base y los resultados chilenos expresados en puntajes directos no normativos.

\section{Método}

\section{Participantes}

El grupo de estudio, mujeres adolescentes infractoras de ley, está constituido por 30 jóvenes de entre 13 y 18 años de edad, encausadas judicialmente por diferentes delitos. Sus antecedentes y protocolos MACI han sido recolectados por el equipo de investigación en un período de aproximadamente cinco años y no habían sido analizados hasta ahora. El primer grupo de contraste, hombres adolescentes infractores de ley, ha sido extraído de una muestra mayor $(n=151)$ recolectada en el mismo período y no utilizada previamente; este grupo consta de 30 participantes. Para su extracción se controló la edad de los adolescentes y parcialmente sus años de educación pareándolos con las adolescentes en estudio. El segundo grupo de contraste, mujeres adolescentes con problemas de salud mental, fue extraído de una muestra de adolescentes consultantes por problemas de salud mental no se externalizados en desadaptación social $(n=168)$; este grupo consta de 30 adolescentes. Para su extracción desde la muestra mayor se controló la edad de las participantes para hacerla equivalente al grupo de mujeres adolescentes infractoras de ley.

\section{Procedimiento}

Con la aprobación del estudio por el Comité de Ética de la universidad y del servicio de salud correspondiente, los y las adolescentes fueron contactados en sus centros de atención psicológica; allí se les solicitó su participación voluntaria luego de informarles sobre los objetivos del estudio y asegurarles la confidencialidad de la información. Quienes aceptaron participar firmaron un consentimiento informado y contestaron el MACI bajo las instrucciones estándares en grupos pequeños o individualmente; sus protocolos fueron codificados en puntajes directos y convertidos a puntajes normativos según las normas chilenas (Vinet \& Forns, 2008). Además, para los y las adolescentes infractores se desarrolló una entrevista individual con los educadores o profesionales responsables de su cuidado y un análisis documental de fuentes secundarias; este procedimiento significó aproximadamente dos horas de trabajo adicional por cada adolescente y permitió completar un registro de escalada de desadaptación social y eventos de riesgo, denominado Ficha de Escalada y Riesgo (FER).

\section{Instrumentos y medidas}

(1) Inventario Clínico para Adolescentes de Millon (MACI) (Millon, 1993). Es un instrumento de construcción teórica que sigue los modelos de aprendizaje biosocial y evolutivo de la personalidad desarrollados por Millon (1969, 1990) y que consta de 160 ítems con formato VerdaderoFalso. Los ítems se organizan en 31 escalas, 27 de ellas con significado clínico, estas son doce escalas de Patrones de Personalidad, ocho escalas de Preocupaciones Expresadas y siete escalas de Síndromes Clínicos. Las escalas no clínicas son tres escalas de control o Escalas Modificadoras y una escala de validez (V) de sólo dos ítems. La denominación de cada escala, según la versión chilena, aparece en la primera columna de la Tabla 3 y siguientes.

La fiabilidad de las escalas ha sido probada en diversos estudios. En muestras chilenas los índices de consistencia interna obtenidos a través del coeficiente Alfa de Cronbach son similares a los obtenidos por Millon (1993) y oscilan entre .54 (D) y .90 (8B) en las muestras de nopacientes y entre .51 (D) y .91 (8B y B) en las muestras de sujetos con problemas psicológicos, con medianas de $.79 \mathrm{y}$ .81 respectivamente (Vinet \& Alarcón, 2003). El instrumento ha demostrado ser una prueba válida, con una adecuada capacidad de discriminación entre adolescentes normales y grupos con problemas psicológicos (Vinet \& Alarcón, 2003) y con buenas posibilidades para caracterizar psicológicamente a diversos grupos en riesgo (Vinet, Salvo, \& Forns, 2005), con problemas clínicos (Vinet \& Alarcón, 2003) y de desadaptación social (Alarcón e cols., 2005).

El MACI cuenta actualmente con tres grupos de normas. Las dos primeras (estadounidenses y españolas) están expresadas en puntajes de Tasa Base asociados a tasas de prevalencia de trastornos mentales y evalúan diferenciadamente a hombres y mujeres de 13 a 15 años y de 16 a 19 años. 
El tercer grupo son las normas chilenas en Puntajes Transformados con Puntos de Corte desarrolladas recientemente por Vinet y Forns (2008), ellas incluyen baremos diferenciados por sexo para adolescentes entre 13 y 19 años de edad.

Las normas chilenas poseen dos características que las distinguen de las otras normas. En primer lugar, integran información sobre la capacidad de discriminación de las escalas entre no-consultantes y consultantes y la dirección ascendente o descendente de los puntajes indicativos de patología en el contexto cultural latinoamericano, agrupando las escalas del MACI en tres categorías: escalas Acordes con la Teoría (AT), escalas Contra-Teóricas (CT) y escalas No Diferenciadoras entre no-consultantes y consultantes (ND). Este último grupo de escalas sólo aparece en las normas de mujeres e incluye a tres de las 27 escalas con significado clínico, ellas son Insensibilidad Social, Predisposición a la Delincuencia y Sentimientos de Ansiedad. En segundo lugar, las normas chilenas del MACI están expresadas en una escala de medida que aúna criterios dimensionales y categoriales a través de la presencia de un puntaje central (PT 50) asociado al punto de corte que, en cada escala, diferencia entre funcionamiento sano y funcionamiento patológico; este puntaje no está presente en las tres escalas ND recién mencionadas.

(2) Ficha de Escalada y Riesgo (FER). Este es un instrumento complejo, de construcción ad-hoc, diseñado por Alarcón (2001), con el objetivo de realizar un riguroso registro de (a) las conductas de desadaptación social en que ha incurrido el o la adolescente y (b) los eventos de riesgo a los que ha estado expuesto. La FER se completa a través de una entrevista desarrollada con los educadores y/o cuidadores y el examen de los expedientes correspondientes a cada adolescente. En este estudio sólo se consideran los elementos más relevantes con el fin de caracterizar a los grupos de mujeres $\mathrm{y}$ varones infractores de ley.

El registro de las conductas de desadaptación social usado en este estudio consta de cuatro categorías: delitos contra las personas, delitos contra la propiedad o las cosas, delitos sexuales y desajustes conductuales. Cada categoría está compuesta por diferentes conductas específicas diferenciadas según gravedad; estas se puntúan con ponderaciones según frecuencia de ocurrencia y dan lugar, en cada categoría, a un índice compuesto. La suma total del puntaje asignado a las categorías compone el Índice de Escalada de Desadaptación Social (IEDS) el cual es una medida global de desadaptación.

El registro de eventos de riesgo usado en este estudio distingue entre riesgo conductual y riesgo psicosocial. El riesgo conductual corresponde a indicadores de conducta explícita del adolescente detectados en la interacción con los sistemas policial y judicial, los cuales son sancionados como conducta desadaptativa en el sistema judicial chileno. Los indicadores de riesgo conductual incluyen la edad de inicio de la conducta desadaptativa, los años de inadaptación, y el número de ingresos a instituciones de diagnóstico o rehabilitación del Servicio Nacional de Menores. Todos ellos son computados en su valor real. El riesgo psicosocial corresponde a indicadores sobre la historia y condiciones de vida del adolescente que han provocado o provocan actualmente un impacto negativo en su adaptación social. Las categorías incluidas son desajuste escolar, relación con pares desadaptados, desestructuración familiar, modelos familiares inadecuados y violencia intrafamiliar. En cada categoría, los indicadores específicos son tabulados como presencia o ausencia y ponderados según gravedad con el fin de obtener un índice compuesto por categoría; la suma total de los valores parciales da lugar al Índice de Riesgo Psicosocial (IRPS) que es una medida global de factores de riesgo.

\section{Análisis de datos}

La información recabada con la FER fue codificada cuantitativamente para permitir la comparación de grupos. Los protocolos MACI fueron puntuados según las normas chilenas (Vinet \& Forns, 2008). Las comparaciones de grupos se realizaron a través de la prueba $Z$ de diferencias entre proporciones independientes de Guilford (1965) para los datos no-paramétricos y a través de pruebas $t$ de Student para los datos paramétricos. Las escalas ND del MACI fueron analizadas a través de la prueba $Z$ de Guilford pues están expresadas en percentiles.

\section{Resultados}

A continuación se presenta una caracterización general de las jóvenes infractoras y se determinan sus diferencias con respecto a los varones de la misma condición. Seguidamente, se exponen los resultados del MACI, a través de contrastes entre mujeres y varones infractores, y luego, entre las adolescentes infractoras y sus pares con problemas clínicos.

\section{Caracterización deadolescentes infractoras en comparación con varones infractores}

La edad media de mujeres y hombres infractores fue de 15.5 años y su educación alcanzó a 7.8 años de instrucción. El computo de la causa por la cual fueron procesados (Tabla 1) muestra que los hombres cometen significativamente más delitos contra las personas y que en las adolescentes predominan los delitos contra la propiedad y los desajustes conductuales.

Tabla 1

Causas de procesamiento de los adolescentes infractores según sexo

\begin{tabular}{lccccc}
\hline \multirow{1}{*}{ Causa } & \multicolumn{3}{c}{ Hombres } & \multicolumn{4}{c}{ Mujeres } & \multirow{2}{*}{$\mathbf{Z}$} \\
& $F$ & $\%$ & $F$ & $\%$ & \\
\hline Delitos Contra Personas & 9 & 30.0 & 1 & 3.3 & $2.77^{* *}$ \\
Delitos Contra Propiedad & 14 & 46.7 & 10 & 33.3 & 1.05 \\
Delitos Sexuales & 2 & 6.7 & 4 & 13.3 & -0.86 \\
Desajustes Conductuales & 3 & 10.0 & 9 & 30.0 & -1.94 \\
Abuso de Substancias & 2 & 6.7 & 6 & 20.0 & -1.52 \\
\hline
\end{tabular}


En los indicadores provistos por la FER - desadaptación social y riesgo psicosocial - se observa que los hombres obtienen un IEDS global mayor que las mujeres, con diferencias significativas en los índices de delitos contra la propiedad y desajustes conductuales. En el riesgo psicosocial se observa que el IRPS global es significativamente más alto en las mujeres, lo mismo sucede con el indicador parcial de violencia intrafamiliar (Tabla 2). Estas diferencias permiten plantear que la desadaptación social presente en los varones es de mayor gravedad, frecuencia y reincidencia; por su parte, las mujeres estarían sometidas a más condiciones de riesgo psicosocial, destacando su vulnerabilidad ante la violencia intrafamiliar. Además, es interesante observar que los factores de riesgo - relación con pares, desajuste escolar, desestructuración familiar y modelos familiares inadecuados -, no difieren de modo significativo en los dos grupos de adolescentes infractores, corroborando que están presentes con la misma intensidad en ambos sexos.

Tabla 2

Índices de desadaptación social y riesgo psicosocial según sexo

\begin{tabular}{lcccccc}
\hline \multicolumn{1}{c}{ IEDS y IRPS } & \multicolumn{2}{c}{ Hombres } & \multicolumn{2}{c}{ Mujeres } & \multirow{2}{*}{$t$} \\
& $M$ & $D T$ & $M$ & $D T$ & & \\
\hline Delitos contra personas & 2,10 & 4,38 & 1,00 & 2,32 & 1,22 & \multirow{2}{*}{ Den } \\
Delitos contra propiedad & 9,70 & 7,97 & 4,43 & 6,02 & 2,89 & $* *$ \\
Delitos sexuales & 0,40 & 1,22 & 0,37 & 0,93 & 0,12 & $*$ \\
Desajustes conductuales & 2,15 & 1,74 & 3,33 & 2,25 & $-2,28$ & $*$ \\
Escalada de Desadaptación Social & 14,35 & 11,12 & 9,13 & 8,00 & 2,09 & $*, 17$ \\
Desajuste escolar & 2,05 & 1,15 & 2,00 & 1,10 & 0,17 \\
Relación con pares desadaptados & 3,13 & 1,49 & 3,00 & 1,54 & 0,34 & \\
Desestructuración familiar & 3,75 & 2,03 & 4,30 & 2,15 & $-1,02$ & \\
Modelos familiares inadecuados & 1,17 & 1,21 & 1,50 & 1,36 & $-1,01$ & $* *$ \\
Violencia intrafamiliar & 1,40 & 1,57 & 3,23 & 1,68 & $-4,38$ & $* *$ \\
Riesgo Psico-Social & 11,50 & 4,43 & 14,03 & 5,16 & $-2,04$ & $*$ \\
\hline
\end{tabular}

${ }^{* * *} p<0.001,{ }^{* *} p<0.01,{ }^{*} p<0.05$

\section{Caracterización a través del MACI de adolescentes infractores según sexo}

Los resultados correspondientes a la comparación entre hombres y mujeres infractores se presentan en la Tabla 3 para todas las escalas, con excepción de las tres escalas ND (Insensibilidad Social, Predisposición a la Delincuencia y Sentimientos de Ansiedad), en ellas, al usar baremos en percentiles no es posible establecer una comparación a través de pruebas $t$.

Primeramente, se observa que todos los valores obtenidos, en los dos grupos, son valores clínicos, ubicados por sobre el puntaje de corte (PT 50); la única excepción es la escala Desaprobación Corporal que presenta valores inferiores. En segundo lugar, se aprecia que en todas las escalas informadas, los valores obtenidos por las adolescentes infractoras son mayores que los obtenidos por sus pares varones; sin embargo, se observan diferencias estadísticamente significativas sólo en tres escalas (Poderoso, Conformista e Inseguridad Grupal) y diferencias marginalmente significativas en las escalas Afligido ( $p=.075)$ y Tendencia Límite $(p=.054)$.

La interpretación conjunta de estas escalas describe a una adolescente infractora que se presenta hostil y combativa, que muestra indiferencia hacia las consecuencias destructivas o displacenteras de sus actos, que no es capaz de respetar las normas sociales ni las expectativas de los otros, especialmente si se trata de adultos en posiciones de autoridad. Aparentemente, disfruta con la dominación, la intimidación y el control agresivo de otros; paradójicamente, presenta tristeza y preocupación por el rechazo de sus pares, temor al ridículo $\mathrm{y}$, posiblemente, fuertes sentimientos de pérdida o abandono de las figuras de apego más significativas. En este funcionamiento dual, las oscilaciones emocionales y las conductas impredecibles son las características más compatibles con un futuro estilo límite de personalidad.

\section{Comparación a través del MACI entre las adolescentes infractoras y las adolescentes con problemas de salud mental}

Las adolescentes infractoras y las adolescentes con problemas clínicos tienen la misma media de edad (15.5 años); sin embargo, las adolescentes infractoras presentan un nivel de escolaridad significativamente menor $\left(M_{\text {Infractoras }}=7.77\right.$, $\left.D T=1.81 ; M_{\text {Clinicas }}=9.43, D T=1.63 ; t=-3.73, p<.001\right)$. Los resultados correspondientes a la comparación entre los dos grupos en las escalas MACI se presentan en la Tabla 4.

Aunque se observa, en ambos grupos, un gran número de escalas con puntuaciones clínicas sobre PT 50, las puntuaciones más elevadas aparecen en las adolescentes infractoras. Las elevaciones del perfil de las adolescentes infractoras indican la acentuación de un patrón de aflicción, que refleja la dificultad para disfrutar y un predominio de dolor psicológico y estrés; estas características están también presentes en las adolescentes de consulta clínica pero en ellas aparecen con menor intensidad. Las diferencias estadísticamente significativas se presentan en siete de las 27 escalas clínicas. 
Tabla 3

Estadísticos descriptivos del MACI y contraste según sexo en adolescentes infractores de ley

\begin{tabular}{|c|c|c|c|c|c|c|c|}
\hline \multirow{2}{*}{\multicolumn{2}{|c|}{ Escalas }} & \multicolumn{2}{|c|}{ Hombres } & \multicolumn{2}{|c|}{ Mujeres } & \multirow{2}{*}{\multicolumn{2}{|c|}{$t$}} \\
\hline & & $M$ & $D T$ & $M$ & $D T$ & & \\
\hline 1 & Introvertido & 52,77 & 13,27 & 58,63 & 12,56 & $-1,76$ & \\
\hline $2 \mathrm{~A}$ & Inhibido & 53,93 & 12,01 & 59,27 & 14,68 & $-1,54$ & \\
\hline $2 \mathrm{~B}$ & Afligido & 60,53 & 18,25 & 69,17 & 18,59 & $-1,82$ & \\
\hline 3 & Sumiso & 51,90 & 10,82 & 55,77 & 10,82 & $-1,38$ & \\
\hline 4 & Dramatizador & 50,80 & 11,62 & 54,70 & 13,90 & $-1,18$ & \\
\hline 5 & Egoísta & 54,23 & 13,67 & 54,50 & 12,98 & $-0,08$ & \\
\hline $6 \mathrm{~A}$ & Trasgresor & 62,27 & 13,07 & 64,30 & 10,88 & $-0,66$ & \\
\hline $6 \mathrm{~B}$ & Poderoso & 51,90 & 18,07 & 62,40 & 14,02 & $-2,51$ & $*$ \\
\hline 7 & Conformista & 55,23 & 13,14 & 62,87 & 11,37 & $-2,41$ & $*$ \\
\hline $8 \mathrm{~A}$ & Oposicionista & 57,70 & 15,06 & 62,00 & 14,51 & $-1,13$ & \\
\hline $8 \mathrm{~B}$ & Autodegradante & 57,13 & 18,48 & 62,70 & 16,50 & $-1,23$ & \\
\hline 9 & Tendencia Limítrofe & 61,07 & 19,49 & 70,80 & 18,59 & $-1,98$ & \\
\hline A & Difusión de la Identidad & 58,57 & 15,57 & 62,37 & 14,39 & $-0,98$ & \\
\hline $\mathrm{B}$ & Autodevaluación & 59,70 & 17,73 & 63,57 & 18,56 & $-0,83$ & \\
\hline $\mathrm{C}$ & Desaprobación Corporal & 47,83 & 19,43 & 47,27 & 25,19 & 0,10 & \\
\hline $\mathrm{D}$ & Incomodidad Sexual & 51,20 & 12,50 & 55,97 & 10,07 & $-1,63$ & \\
\hline $\mathrm{E}$ & Inseguridad Grupal & 50,37 & 12,55 & 61,43 & 16,65 & $-2,91$ & $* *$ \\
\hline $\mathrm{F}$ & Insensibilidad Social & 52,37 & 12,82 & - & - & - & \\
\hline $\mathrm{G}$ & Discordia Familiar & 57,17 & 11,78 & 63,27 & 15,52 & $-1,72$ & \\
\hline $\mathrm{H}$ & Abuso Infantil & 61,63 & 10,47 & 66,07 & 15,32 & $-1,31$ & \\
\hline $\mathrm{AA}$ & Disfunciones Alimentarias & 53,40 & 10,10 & 50,47 & 23,22 & 0,64 & \\
\hline BB & Tendencia Abuso Sustancias & 65,50 & 14,17 & 68,00 & 10,567 & $-0,78$ & \\
\hline $\mathrm{CC}$ & Predisposición Delincuencia & 63,63 & 11,84 & - & - & - & \\
\hline DD & Tendencia a la Impulsividad & 60,10 & 16,49 & 65,93 & 14,43 & $-1,46$ & \\
\hline $\mathrm{EE}$ & Sentimientos de Ansiedad & 57.10 & 13.29 & - & - & - & \\
\hline $\mathrm{FF}$ & Afecto Depresivo & 54,23 & 17,56 & 61,63 & 17,91 & $-1,62$ & \\
\hline GG & Tendencia Suicida & 57,50 & 20,63 & 66,00 & 21,17 & $-1,58$ & \\
\hline
\end{tabular}

Nota. Los valores no informados corresponden a las escalas No Diferenciadoras de los baremos de mujeres los cuales están expresados en percentiles. ${ }^{* * *} p<0.001,{ }^{* *} p<0.01,{ }^{*} p<0.05$

Tabla 4

Estadísticos descriptivos del MACI y contraste entre adolescentes infractoras de ley y adolescentes con problemas clínicos

\begin{tabular}{|c|c|c|c|c|c|c|c|}
\hline \multirow{2}{*}{\multicolumn{2}{|c|}{ Escalas }} & \multirow{2}{*}{\multicolumn{2}{|c|}{ Infractoras }} & \multirow{2}{*}{\multicolumn{2}{|c|}{ Clínicas }} & \multirow{2}{*}{$t$} & \multirow{2}{*}{$t$} \\
\hline & & & & & & & \\
\hline 1 & Introvertido & 5863 & 1256 & 5637 & 1321 & 068 & \\
\hline $2 \mathrm{~A}$ & Inhibido & 59,27 & 14,68 & 55,70 & 15,53 & 0,91 & \\
\hline $2 \mathrm{~B}$ & Afligido & 69,17 & 18,59 & 61,67 & 24,97 & 1,32 & \\
\hline 3 & Sumiso & 55,77 & 10,82 & 50,80 & 13,91 & 1,54 & \\
\hline 4 & Dramatizador & 54,70 & 13,90 & 54,60 & 13,44 & 0,03 & \\
\hline 5 & Egoísta & 54,50 & 12,98 & 54,63 & 15,52 & $-0,04$ & \\
\hline $6 \mathrm{~A}$ & Trasgresor & 64,30 & 10,88 & 53,43 & 15,84 & 3,10 & $* *$ \\
\hline $6 \mathrm{~B}$ & Poderoso & 62,40 & 14,02 & 52,30 & 18,12 & 2,41 & $*$ \\
\hline 7 & Conformista & 62,87 & 11,37 & 60,10 & 16,22 & 0,77 & \\
\hline $8 \mathrm{~A}$ & Oposicionista & 62,00 & 14,51 & 58,47 & 15,61 & 0,91 & \\
\hline $8 B$ & Autodegradante & 62,70 & 16,50 & 58,83 & 21,21 & 0,79 & \\
\hline 9 & Tendencia Limítrofe & 70,80 & 18,59 & 62,60 & 24,68 & 1,45 & \\
\hline $\mathrm{A}$ & Difusión de la Identidad & 62,37 & 14,39 & 57,97 & 17,62 & 1,06 & \\
\hline $\mathrm{B}$ & Autodevaluación & 63,57 & 18,56 & 59,63 & 22,00 & 0,75 & \\
\hline $\mathrm{C}$ & Desaprobación Corporal & 47,27 & 25,19 & 51,60 & 27,14 & $-0,64$ & \\
\hline $\mathrm{D}$ & Incomodidad Sexual & 55,97 & 10,07 & 54,13 & 11,81 & 0,65 & \\
\hline
\end{tabular}


Tabla 4 - Continuación

\begin{tabular}{|c|c|c|c|c|c|c|c|}
\hline \multirow{2}{*}{\multicolumn{2}{|c|}{ Escalas }} & \multirow{2}{*}{\multicolumn{2}{|c|}{ Infractoras }} & \multirow{2}{*}{\multicolumn{2}{|c|}{ Clínicas }} & \multirow{2}{*}{$t$} & \\
\hline & & & & & & & \\
\hline & & 112 & $16 c^{-}$ & 50 & ר20 & 18 & \\
\hline & & 01,43 & 10,03 & 52,00 & 20,32 & 1,84 & \\
\hline $\mathrm{G}$ & Discordia Familiar & 63,27 & 15,52 & 58,23 & 16,93 & 1,20 & \\
\hline $\mathrm{H}$ & Abuso Infantil & 66,07 & 15,32 & 52,30 & 19,88 & 3,01 & $* *$ \\
\hline $\mathrm{AA}$ & Disfunciones Alimentarias & 50,47 & 23,22 & 49,70 & 24,52 & 0,12 & \\
\hline BB & Tendencia Abuso Sustancias & 68,00 & 10,57 & 50,67 & 16,46 & 4,85 & $* * *$ \\
\hline DD & Tendencia a la Impulsividad & 65,93 & 14,43 & 59,00 & 17,96 & 1,65 & \\
\hline FF & Afecto Depresivo & 61,63 & 17,91 & 60,00 & 20,83 & 0,33 & \\
\hline \multirow[t]{2}{*}{ GG } & Tendencia Suicida & 66,00 & 21,17 & 58,57 & 25,58 & 1,23 & \\
\hline & Escalas No Diferenciadoras & $M d_{\text {Infractoras }}$ & & $M d_{\text {Clinicas }}$ & & $Z$ & \\
\hline $\mathrm{F}$ & Insensibilidad Social & 78,50 & & 59,00 & & $-2,18$ & $*$ \\
\hline $\mathrm{CC}$ & Predisposición Delincuencia & 96,00 & & 66,00 & & $-3,11$ & $* *$ \\
\hline EE & Sentimientos de Ansiedad & 20,00 & & 39,00 & & $-1,99$ & $*$ \\
\hline
\end{tabular}

Nota: Las escalas No Diferenciadoras están baremadas en percentiles.

${ }^{* * *} p<0.001,{ }^{* *} p<0.01,{ }^{*} p<0.05$

A nivel de Patrones de la Personalidad; la elevación conjunta en las escalas Trasgresor y Poderoso indica la presencia de trastornos conductuales y un actuar oposicionista o antisocial; hay un estilo de comportamiento hostil con rechazo de las normas sociales, crueldad en las relaciones interpersonales y dificultad para sentir culpa genuina o remordimiento. En el ámbito de las Preocupaciones estas adolescentes presentan frialdad e indiferencia con respecto a los sentimiento y reacciones de los otros (escala F; ver en la parte baja de la Tabla), sin embargo, al mismo tiempo se observa que la inseguridad y tristeza ante el posible rechazo de sus pares (escala E) es alta y marginalmente significativa $(p=.075)$. Estas características coexisten con sentimientos disfóricos de vergüenza y/o disgusto en relación a experiencias de maltrato o abuso (escala $\mathrm{H}$ ) infligidas generalmente por personas significativas cercanas (padres, parientes, hermanos o amigos). Finalmente, las elevaciones en las escalas Abuso de Sustancias y Predisposición a la Delincuencia, junto a la baja en Sentimientos de Ansiedad, indican que estas adolescentes presentan trastornos conductuales caracterizados por impulsividad, actuaciones defensivas y desprecio por las normas sociales y un menor registro de sentimientos ansiosos, lo cual es compatible con mayores conductas de riesgo y conducta delictiva.

El perfil descrito se presenta gráficamente en la Figura 1. $\mathrm{Al}$ contrastarlo con los perfiles de la muestra de baremación correspondientes a las no consultantes (ubicado en el área inferior del gráfico) y las consultantes (ubicado en el área superior del gráfico), se aprecian elevaciones en los patrones de la personalidad que destacan el carácter límite que el perfil de las jóvenes infractoras puede adquirir. Así mismo, destacan las elevaciones de las últimas cuatro escalas de Preocupaciones Expresadas (E, F, G y H) que señalan una actitud ambivalente hacia las relaciones interpersonales junto a disfunción familiar y probables experiencias de abuso en la infancia. En Síndromes Clínicos el conjunto formado por las puntuaciones de BB, CC, DD y EE representa la especificidad del estilo de funcionamiento delictual.

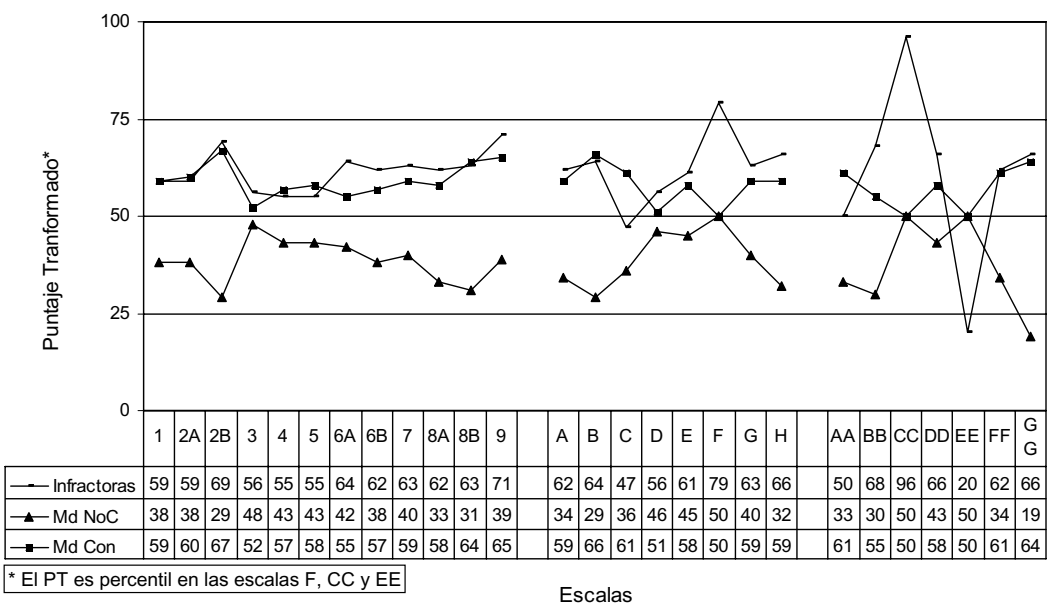

Figura 1. Perfil grupal del MACI de 30 mujeres infractoras de ley. 


\section{Discusión}

La caracterización general de las adolescentes infractoras es compatible con la caracterización elaborada por Mullis e cols. (2004). En comparación con los varones infractores, sus delitos son menos violentos (IEDS menor); sin embargo su nivel de riesgo es mayor (IRPS más alto, con elevada presencia de violencia intrafamiliar) coincidiendo con lo reportado por Dell'Aglio e cols. (2005). En comparación con sus pares clínicas tienen un menor nivel de escolaridad y mayor vulnerabilidad.

En el MACI, aunque no se observa una correspondencia exacta con los estudios de Loper e cols. (2001) y Alarcón (2001), el perfil general de las adolescentes delincuentes (Figura 1) muestra un grupo de elevaciones clínicas en las escalas de Patrones de la Personalidad que indican trastornos internalizados (Introvertido, Inhibido, Afligido, Autodegradante) que coexiste con un grupo de elevaciones en escalas que representan funcionamiento delincuencial (Trasgresor, Poderoso, No-conformista, Oposicionista). Esto posibilita las grandes oscilaciones afectivo-emocionales y las conductas disruptivas e impredecibles propias de las adolescentes que se involucran en comportamientos delictivos. A nivel de Preocupaciones se observa la actitud contradictoria hacia los otros (elevación conjunta de Inseguridad Grupal e Insensibilidad Social), la vulnerabilidad familiar (G) y la presencia de abuso infantil (H). En Síndromes Clínicos, el perfil propio de los trastornos delincuenciales (BB, CC, DD y bajo EE) coexiste con elevaciones clínicas en Afecto Depresivo y Tendencia Suicida.

Este perfil permite entender muchas de las características que han sido señaladas en diferentes estudios descriptivos sobre adolescentes delincuentes como los sentimientos disfóricos ante la vida y la carencia de esperanza en el futuro; las experiencias de abuso y explotación, muchas veces perpetradas por familiares cercanos (Mullis e cols., 2004); la baja autoestima y la alta incidencia de conductas suicidas (McCabe e cols., 2002), y la mayor prevalencia de trastornos mentales en general (Timmons-Mitchell e cols., 1997; Cruise e cols., 2007). Además, tal como lo señalan Andrade e cols. (2004), en este grupo coexisten los trastornos internalizados junto a manifestaciones definidamente externalizantes como la conducta transgresora. El perfil descrito integraría características de los tipos "Problemas Externalizados" y "Depresivas Interpersonalmente Ambivalentes" encontrados por Stefurak y Calhoun (2007); sin embargo, como se trata de una muestra pequeña, no es posible detectar la presencia de subtipos tal como estas autoras lo hicieran. Adicionalmente, es importante señalar que aunque los resultados obtenidos, expresados en Puntajes Transformados con Puntos de Corte, no presentan una correspondencia escala a escala con los estudios precedentes, entregan un perfil que integra los elementos más relevantes de esos estudios.

\section{Conclusiones}

Este estudio contribuye a la visibilización de las características de las adolescentes infractoras destacando sus similitudes y diferencias con respecto a los varones infractores. Se observa que ambos grupos de adolescentes han estado igualmente expuestos a factores de riesgo ampliamente descritos en estudios sobre reincidencia como su relación con pares con compromiso delictivo, desajuste escolar, desestructuración familiar y modelos familiares vinculados a la vida delictiva. Sin embargo, el grupo femenino muestra claramente una mayor exposición a la victimización en el medio familiar a través de violencia, maltrato y/o abuso sexual, desarrollando necesidades psicológicas que se expresan en sus comportamientos desafiantes y antisociales.

El análisis a través del MACI confirma las características de personalidad adolescente descritas en estudios previos en varones infractores y en grupos mixtos. El análisis específico de las jóvenes infractoras aporta la presencia de un funcionamiento dual donde coexisten estilos de personalidad vinculados a la trasgresión junto a estilos relacionados con la aflicción y vulnerabilidad emocional. Esta información permitiría diseñar estrategias de intervención diferenciada al evaluar, en las adolescentes, el actuar delictual junto a las necesidades psicológicas y el grado de daño en la victimización. Se plantea que algunas adolescentes podrían responder a intervenciones basadas en reparación de vínculos emocionales considerando variables personales y familiares, en tanto que otras, con una predisposición delictual sin correlato emocional de daño y/o inmersas en contextos de alto riesgo criminogénico, requerirían de intervenciones más focalizadas en los aspectos conductuales del comportamiento trasgresor. Además, al comparar a estas adolescentes con sus pares consultantes por trastornos clínicos destaca la mayor gravedad de sus alteraciones en salud mental; este hallazgo complejiza la posibilidad de cambio a través de intervenciones breves y no especializadas destacando la necesidad de detección e intervención precoz y la aplicación de programas de intervención diferenciados e intensivos donde el trabajo con los vínculos (familia, pareja) no puede quedar marginado del foco de la intervención.

El MACI, como instrumento de evaluación, demuestra su sensibilidad para detectar las características diferenciales del grupo estudiado, tanto a nivel de estilos de funcionamiento como de necesidades psicológicas específicas. Además, es importante señalar que las escalas Insensibilidad Social, Predisposición a la Delincuencia y Sentimientos de Ansiedad, categorizadas como No Diferenciadoras en la baremación chilena de mujeres (Vinet \& Forns, 2008), aportan a una diferenciación significativa entre las adolescentes con comportamiento antisocial y sus pares con trastornos clínicos, siendo necesario continuar su estudio para afinar su significado interpretativo en este tipo de muestras. 


\section{Referencias}

Alarcón, P. (2001). Evaluación psicológica de adolescentes con desadaptación social: Un estudio a través del MACI e indicadores de riesgo en el sur de Chile. Tesis de master no publicada, Universidad de Salamanca, España.

Alarcón, P., Vinet, E., \& Salvo, S. (2005). Estilos de personalidad y desadaptación social durante la adolescencia. Psykhe, 14(1), 3-16.

Andrade, R. C., Silva, V. A., \& Assumpção, F. B., Jr. (2004). Preliminary data on the prevalence of psychiatric disorders in Brazilian male and female juvenile delinquents. Brazilian Journal of Medical and Biological Research, 37, 1155-1160.

Corporación Chilena Pro Derechos de los Niños y los Jóvenes. (2006). Estudio: Género y adolescentes infractores de ley: Informe final. Recuperado en 28 noviembre 2007, de http://www.sename.cl/SenameWebNeo/Controls/ Neochannels/Neo_CH6263/deploy/estudio_genero_ infractores_sename.pdf

Cruise, K., Marsee, M., Dandreaux, D., \& DePrato, D. (2007). Mental health screening of female juvenile offenders: Replication of a subtyping strategy. Journal of Child and Family Studies, 16, 615-625.

Dell'Aglio, D. D., Benetti, S. P., Cruz, S., Deretti, L., Bergesch, D., \& Severo, L. J. (2005). Eventos estressores no desenvolvimento de meninas adolescentes cumprindo medidas sócio-educativas. Paideia, 15, 119-129.

Dixon, A., Howie P., \& Starling, J. (2004). Psychopathology in female juvenile offenders. Journal of Child Psychology and Psychiatry, 45, 1150-1158.

Emler, N., \& Reicher, S. (1995). Adolescence and delincuency. Oxford, UK: Blackwell Pub.

Guilford, J. P. (1965). Fundamental statistics in psychology and education. New York: McGraw-Hill.

Juliano, D. (2008). De la sartén a las brasas: Riesgo, delito $y$ pecado femenino. Trabajo presentado en $10^{\circ}$ Congreso Internacional Interdisciplinar sobre las Mujeres, Mundos de Mujeres/Women's Worlds, Universidad Complutense de Madrid, España.

Lenssen, S. A., Doreleijers, T. A., Van Dijk, M. E., \& Hartman, C. A. (2000). Girls in detention: What are their characteristics? A project to explore and document the character of this target group and the significant ways in which it differs from one consisting of boys. Journal of Adolescence, 23, 287-303.

Loper, A. B., Hoffschmidt, S. J., \& Ash, E. (2001). Personality features and characteristics of violent events committed by juvenile offenders. Behavioral Sciences and the Law, 19, 81-96.

McCabe, K. M., Lansing, A. E., Garland A., \& Hough, R. (2002). Gender differences in psychopathology, functional impairment, and familial risk factors among adjudicated delinquents. Journal of the American Academy of Child and Adolescent Psychiatry, 41, 860-868.
Millon, T. (1969). Modern psychopathology: A biosocial approach to maladaptative learning and functioning. Philadelphia, PA: Saunders.

Millon, T. (1990). Toward a newpersonology: An evolutionary model. New York: Wiley.

Millon, T. (1993). Manual of millon adolescent clinical inventory. Minneapolis, MN: Nacional Computer Systems.

Moffitt, T. E. (1993). Adolescence-limited and lifecourse persistent antisocial behavior: A developmental taxonomy. Psychological Review, 100, 674-701.

Mullis, R. L., Cornille, T. A., Mullis, A. K., \& Huber, J. (2004). Female juveline offending: A review of characteristics and contexts. Journal of Child and Family Studies, 13, 205-218.

Musitu, G., Buelga, S., Lila M., \& Cava, M. (2001). Familia $y$ adolescencia. Madrid: Sintésis.

Navarro, V. (2005). Obligaciones internacionales de México en materia de justicia penal adolescente: Las niñas y adolescentes en conflicto con la ley: Una mirada sociológica. Trabajo presentado en Seminario Internacional sobre los Derechos Humanos de los Niños, Niñas y Adolescentes. Recuperado en 02 Julio 2008, de http://portal.sre.gob.mx/pcdh/libreria/libro7/10\%20F.pdf

Odgers, C., Moretti, M. L., Burnette, M., Chauhan, P., Waite, D., \& Rapocci, D. (2007). A latent variable modeling approach to identifying subtypes of serious and violent female juvenile offenders. Aggressive Behavior, 33, 339-352.

Servicio Nacional de Menores. (2007). Sistema nacional de atención socioeducativo para adolescentes infractores de ley: Periodo 2006-2010. Gobierno de Chile. Recuperado en 28 noviembre 2007, de http://www.sename.cl/ SenameWebNeo/Controls/Neochannels/Neo_CH6186/ deploy/Siistema_nacional.pdf

Silverthorn, P., \& Frick, P. J. (1999). Developmental pathways to antisocial behavior: The delayed-onset pathway in girls. Development and Psychopathology, 11, 101-126.

Stefurak, T., \& Calhoun, G. B. (2007). Subtypes of female juvenile offenders: A cluster analysis of the Millon adolescent Clinical Inventory. International Journal of Law and Psychiatry, 30, 95-111.

Eugenia Vinet es Profesora Asociada de la Universidad de La Frontera.

Paula Alarcón Bañares es Profesora Asistente de la Universidad de La Frontera. 